\title{
Anti-inflammatory activities of Maillard reaction products from whey protein isolate fermented by Lactobacillus gasseri 4M13 in lipopolysaccharide-stimulated RAW264.7 cells
}

\author{
Da Hyun Kim, ${ }^{1 *}$ Su-Hyun Chun, ${ }^{1 *}$ Nam Su Oh, ${ }^{2}$ Ji Young Lee, ${ }^{1}$ and Kwang-Won Lee ${ }^{1} \dagger$ \\ ${ }^{1}$ Department of Biotechnology, College of Life Sciences and Biotechnology, Korea University, Seoul 02841, Republic of Korea \\ ${ }^{2}$ R\&D Center, Seoul Dairy Cooperative, Ansan, Kyunggi 15407, Republic of Korea
}

\section{ABSTRACT}

Maillard reaction products formed from whey protein isolate (WPI) and sugar have been shown to have an anti-inflammatory effect in vitro. Here, we incubated WPI and galactose (GWA) in an aqueous solution at $65^{\circ} \mathrm{C}$ for $24 \mathrm{~h}$ to produce a glycated conjugate, which was then fermented using Lactobacillus gasseri 4M13 to obtain the fermented product (F-GWA). We demonstrated that F-GWA had an anti-inflammatory effect on lipopolysaccharide (LPS)-stimulated RAW264.7 cells. It reduced both LPS-stimulated nitric oxide production and LPS-stimulated increases in the gene expression levels of tumor necrosis factor- $\alpha$ and cyclooxygenase- 2 in a dose-dependent manner. Furthermore, F-GWA inhibited the LPS-induced phosphorylation of extracellular signal-regulated kinase and c-Jun N-terminal kinase, members of the mitogen-activated protein kinase family. The glycation process was evaluated by measuring fluorescence intensity and the furosine concentration during the Maillard reaction to form GWA. The protein modifications of WPI were analyzed using MALDI-TOF tandem mass spectrometry. We found that the combination of the Maillard reaction and L. gasseri 4M13 fermentation increased the prebiotic properties of GWA as well as organic acid production, compared with the nonreacted WPI and galactose.

Key words: Lactobacillus gasseri 4M13, Maillard reaction, whey protein isolate, galactosylation, antiinflammation

Received September 30, 2018.

Accepted May 28, 2019.

*These authors contributed equally to this work.

†Corresponding author: kwangwon@korea.ac.kr

\section{INTRODUCTION}

Whey contains various immune-related proteins (Marshall, 2004). The majority of whey protein consists of $\beta$-LG, $\alpha-\mathrm{LA}$, and immunoglobulins. Whey protein itself has been reported to have anti-inflammatory effects in various cells, including macrophages (Yamaguchi et al., 2009; Chatterton et al., 2013; Da Silva and Rudkowska, 2015). Storage or heating of whey protein can also lead to its chemical modification. One such modification arises from the Maillard reaction (or glycation reaction), which is a nonenzymatic reaction that occurs between sugars and proteins (van Boekel, 1998). Whey proteins can gain beneficial properties through modification by glycation, including anti-inflammatory effects (Chawla et al., 2009; Wang et al., 2013; Chun et al., 2016; Pyo et al., 2016).

Fermentation by lactic acid bacteria can also provide dairy products with beneficial properties through the production of lactic acid and the subsequent proteolysis of milk proteins (Panesar et al., 2010; Pescuma et al., 2010; Prasad et al., 2014). Lactic acid bacteria produce lactic acid from lactose through the action of $\beta$-galactosidase, and these bacteria are often used as probiotics that can survive in the gastrointestinal tract and provide beneficial health effects (Masood et al., 2011). Fermented dairy products have been reported to have immunomodulatory activities including antitumor (Matar et al., 2001), anti-inflammatory (Merenstein et al., 2010), and immune-enhancing activities (Vinderola et al., 2006; Kang et al., 2017).

In this study, we used Lactobacillus gasseri 4M13 isolated from the feces of healthy infants; this strain has been reported to have anti-inflammatory and antioxidative properties (Oh et al., 2018). Using this Lactobacillus strain, we fermented the glycated conjugate of whey protein isolate (WPI) and galactose (GWA) to produce fermented GWA (F-GWA), and we then demonstrated the anti-inflammatory effect of F-GWA on LPS-stimulated RAW264.7 cells. Chemical characterization of GWA, which was prepared by the reaction 
between WPI and galactose, was carried out to further understand its anti-inflammatory effects.

\section{MATERIALS AND METHODS}

\section{Chemicals and Materials}

Whey protein isolate was purchased from Hilmar Ingredients (Hilmar, CA). Sequencing grade trypsin, isolated from bovine pancreas, was purchased from Roche (Mannheim, Germany). Lipopolysaccharide, isolated from Escherichia coli O111:B4, and sodium nitrite were purchased from Sigma (St. Louis, MO). Dulbecco's modified Eagle's medium (DMEM) with low glucose and nonessential amino acid solution was purchased from Gibco (Carlsbad, CA), and other supplements for media were purchased from HyClone Laboratories Inc. (Logan, UT). Other reagents and materials were purchased as HPLC-or cell culture-grade.

\section{Preparation of Samples}

Preparation of GWA. Glycated conjugate of WPI and galactose was provided by Seoul Dairy Cooperative (Ansan, South Korea). Briefly, WPI was mixed with galactose at a ratio of $2: 1(\mathrm{wt} / \mathrm{wt})$ and dissolved in distilled water to yield a $5 \%$ (wt/vol) solution. The WPIgalactose aqueous solution was incubated at $65^{\circ} \mathrm{C}$ for 24 $\mathrm{h}$ with shaking at $60 \mathrm{rpm}$. Afterward, the solution was dialyzed twice using an ultrafiltration system equipped with a 10,000-Da molecular weight cutoff membrane (Sam Yeon Engineering, Seoul, South Korea) at $50^{\circ} \mathrm{C}$ to eliminate any remaining unreacted material. The dialyzed solution was then lyophilized and stored at $-72^{\circ} \mathrm{C}$.

Preparation of L. gasseri 4M13-Fermented Products. Fermented GWA (F-GWA) was provided by Professor Sae Hun Kim's Laboratory, Korea University. Lactobacillus gasseri 4M13 (R\&D center, Seoul Dairy Cooperative, Ansan, South Korea) was used in this study. The strain was isolated from the feces of healthy newborn Korean infants (Oh et al., 2018). The protein concentration of the lyophilized GWA was measured using the Kjeldahl method. The required amount of galactose was added to reconstitute the protein, using a protein-to-sugar ratio of 2:1 (5\% of the protein in solution) in an aqueous solution. Lactobacillus gasseri $4 \mathrm{M} 13$ $\left(10^{6} \mathrm{cfu} / \mathrm{mL}\right)$ was inoculated into the aqueous GWA solution and incubated for $48 \mathrm{~h}$ at $37^{\circ} \mathrm{C}$ to prepare the F-GWA. As a set of controls, a solution with the same ratio of WPI and galactose but without glycation and a solution with galactose only were also fermented under the same conditions (F-WA, fermented WPI and galactose; F-A, fermented galactose). After fermentation, all solutions were centrifuged at $28,000 \times g$ for $10 \mathrm{~min}$ at $4^{\circ} \mathrm{C}$, and the supernatants were then lyophilized and stored at $-72^{\circ} \mathrm{C}$.

\section{Cell Culture and Sample Treatment}

Cells from the RAW264.7 murine macrophage cell line were purchased from ATCC (Manassas, VA) and cultured in DMEM with $1.5 \mathrm{mg} / \mathrm{mL}$ glucose, $100 \mathrm{U} /$ $\mathrm{mL}$ penicillin, $100 \mathrm{U} / \mathrm{mL}$ streptomycin, nonessential amino acids, and $10 \%$ fetal bovine serum. The cell line was maintained at $37^{\circ} \mathrm{C}$ in a humidified incubator containing $5 \% \mathrm{CO}_{2}$ and subcultured every $2 \mathrm{~d}$, with the medium being changed daily. Cells at passages between 15 and 30 were used for experiments. Samples were adjusted for the amount of protein concentration using a bicinchoninic acid (BCA) protein assay kit (Thermo, Rockford, IL) before use in in vitro experiments.

\section{Measurement of Nitric Oxide Production}

The amount of nitric oxide (NO) released from RAW264.7 cells was measured using the Griess reaction. RAW264.7 cells were plated at a density of $9 \times$ $10^{4}$ cells/well in 96 -well plates. After $24 \mathrm{~h}$, the cells were pretreated for $2 \mathrm{~h}$ with the different samples, and LPS was then added to each well at a final concentration of $100 \mathrm{ng} / \mathrm{mL}$. The plates were then incubated for a further $16 \mathrm{~h}$. For the Griess reaction, supernatants were transferred into a 96-well plate, and Griess reagent [1 part $0.1 \% N$-(1-naphtyl) ethylenediamineand 1 part $5 \%$ phosphoric acid containing $1 \%$ sulfanilamide] was added at a supernatant-to-reagents ratio of 1:2; sodium nitrite was used as the NO standard. The absorbance at $540 \mathrm{~nm}$ was measured using a multiplate reader. The concentration of NO in the medium of each well was calculated from the optical density.

\section{Quantitative Reverse Transcription PCR}

The cells were plated in 6-well plates and then incubated for $24 \mathrm{~h}$. The cells were then pretreated with F-GWA for $2 \mathrm{~h}$, after which LPS was added for a further $4 \mathrm{~h}$. Next, the cells were harvested and incubated with RNAiso plus (Takara, Shiga, Japan), and the total RNA was extracted according to the manufacturer's instructions. The quantities of isolated RNA were determined using a NanoDrop spectrophotometer (Thermo Scientific Inc., Pittsburgh, PA). We used the isolated RNA to synthesize cDNA, using the LeGene Premium Express 1st Strand cDNA synthesis system (LeGene, San Diego, CA) according to the manufacturer's instructions. For mRNA gene expression analysis, quantitative PCR was performed with HiPi Real-time 
SYBR green master mix (Elpis Biotech, Daejeon, South Korea), using an iQ5 Real-time PCR detection system (Bio-Rad, Hercules, CA). All data were analyzed using the $2^{-\Delta \Delta \mathrm{Ct}}$ method (Livak and Schmittgen, 2001) and normalized with $\beta$-actin as the housekeeping gene. The specific Mus musculus mRNA primers used in this study were as follows: $\beta$-actin (housekeeping gene): forward primer, 5'-AGAGGGAAATCGTGCGTGAC-3', reverse primer, 5'-CAATAGTGATGACCTGGCCGT-3'; tumor necrosis factor- $\alpha$ (TNF- $\alpha$ ): forward primer, 5'-CATCTTCTCAAAATTCGAGTGACAA-3', reverse primer, 5'-TGGGAGTAGACAAGGTACAACCC-3'; cyclooxygenase-2 (COX-2), forward primer, 5'-TTCCTCTACATAAGCCAGTGA-3', reverse primer, 5'-TCCACATTACATGCTCCTATC-3'; and IL-10: forward primer, 5'-GGTTGCCAAGCCTTATCGGA-3', reverse primer, 5'-ACCTGCTCCACTGCCTTGCT-3'.

\section{Western Blotting}

To determine the phosphorylation of the mitogenactivated protein kinase (MAPK) signaling proteins, RAW264.7 cells were treated with samples for $2 \mathrm{~h}$, after which LPS was added for a further $30 \mathrm{~min}$ (a total of 150 min of treatment). After treatment, the cells were washed twice with cold PBS and lysed with RIPA buffer (Elpis Biotech) containing a phosphatase inhibitor cocktail (Sigma) and protease inhibitors $(5 \mu \mathrm{g} / \mathrm{mL}$ of leupeptin and aprotinin each; Sigma-Aldrich). After analysis of their protein content with a BCA assay, the cell lysates were adjusted to the same protein concentration and denatured with loading buffer (a final concentration of $2 \%$ SDS) by boiling for $5 \mathrm{~min}$ at $100^{\circ} \mathrm{C}$. The proteins were separated using an SDS-PAGE system and transferred onto polyvinylidene difluoride membranes (Immobilon-P, Millipore, MA). The membranes were blocked for $1 \mathrm{~h}$ at room temperature and incubated with primary antibodies overnight at $4^{\circ} \mathrm{C}$. After being washed and then incubated with secondary antibodies for $45 \mathrm{~min}$ at room temperature, the blots were visualized using a chemiluminescent substrate (SuperSignal, Thermo Fisher, Carlsbad, CA), with the luminescence being captured by film. All antibodies were dissolved in a blocking solution containing $5 \%$ (wt/vol) BSA or nonfat dried milk in Tris-buffered saline containing Tween-20.

\section{Characterization of Samples}

Fluorescence. Samples (a total of 9 samples over 24 h) were collected during glycation for analysis, as previously described (Oh et al., 2017). Briefly, fluorescence was measured at an excitation wavelength of $370 \mathrm{~nm}$ and at an emission wavelength of $440 \mathrm{~nm}$ using a Synergy H1 plate reader (Bio-Tek Instruments Inc., Winooski, VT). To measure changes in advanced Maillard products, the fluorescence intensity was measured at 330- and 420-nm excitation and emission wavelengths, respectively (Guan et al., 2005).

Chemical Composition. The $\varepsilon-N$-2-furoylmethylL-lysine (furosine) concentration was analyzed as previously described (Oh et al., 2013). Briefly, samples were hydrolyzed at high temperature $\left(110^{\circ} \mathrm{C}\right)$ in the presence of acid $(\mathrm{HCl})$ for $24 \mathrm{~h}$. The hydrolysates were prepared using a Sep-Pak C18 cartridge (Waters, Milford, MA), and the elutes were dried with nitrogen gas. Dried samples were analyzed using a HPLC system (Alliance, Waters) with a photodiode array detector set at 280 nm, using a Capcell Pak C18 UG column. Organic acid compositions were analyzed using a method previously reported by Donkor et al. (2005), using an HPLC system (1260, Agilent, Waldbronn, Germany) fitted with an Aminex HPX-87C column (Bio-Rad, Richmond, $\mathrm{CA})$.

MALDI-TOF MS and MS/MS Analysis. To characterize the chemical modification by glycation, protein profiles were analyzed with MALDI-TOF MS/ MS according to a previously reported method (Oh et al., 2017). Trypsin digestion of GWA preceded MALDI-TOF/MS analysis (Bruker Autoflex, Bruker, Germany). From the collected mass spectrometry data, the molecular mass was searched against the nonredundant Swiss-Prot database, using the Mascot search engine (Matrix Science, London, UK). The search was performed against the Bos taurus database or other mammalian databases.

\section{Statistics}

All results are expressed as the mean \pm standard deviation of triplicate samples. Differences between groups were compared using a 1-way ANOVA for repeated measurements followed by Tukey's studentized range test. Statistical analysis was conducted using SAS software version 9.4 (SAS Institute, Cary, NC). A $P$-value of $<0.05$ was used to indicate statistical significance.

\section{RESULTS}

\section{Anti-Inflammatory Effect of F-GWA on RAW264.7 Cells}

To demonstrate the decreased production of NO by RAW264.7 cells after F-GWA treatment, we treated the cells with LPS for $16 \mathrm{~h}$ after they had been pretreated with different concentrations of F-GWA for $2 \mathrm{~h}$. 
Although the amount of NO produced increased after LPS treatment, pretreatment with F-GWA significantly reduced the amount of NO produced $(P<0.05)$ (Figure 1A). In this experiment, the LPS-challenged group showed less viability than the control group (85.4\% of control); however, the pretreatment with F-GWA increased viability (Supplemental Figure S1; https://doi.org/10.3168/jds.2018-15774), indicating no cytotoxicity occurred up to $1,000 \mu \mathrm{g} / \mathrm{mL}$ of F-GWA. The magnitude of this effect increased in a dose-dependent manner; F-GWA treatment showed a significant reduction in NO production $(P<0.05)$ above $100 \mu \mathrm{g} /$ $\mathrm{mL}$. Treatment with $1 \mathrm{mg} / \mathrm{mL}$ F-GWA completely suppressed the NO production due to LPS treatment and returned the NO levels to those of the LPS-untreated control group. Among the different treatments, only the F-GWA-treated cells showed decreased NO production at $100 \mu \mathrm{g} / \mathrm{mL}$ (Figure 1B); WPI and GWA did not inhibit NO production in LPS-treated RAW264.7 cells. From this study, we conclude that both glycation and the subsequent fermentation process are required to produce the NO inhibition effect.

To examine the expression of inflammatory cytokines in F-GWA-treated cells, we used quantitative reverse transcription PCR to measure the mRNA gene expression levels of the pro-inflammatory proteins $T N F-\alpha$ and COX-2. We found that F-GWA significantly reduced the gene expression levels of both TNF- $\alpha$ and COX-2 in a dose-dependent manner (Figure 2). The F-GWA treatment $(100 \mu \mathrm{g} / \mathrm{mL})$ suppressed the COX2 and TNF- $\alpha$ gene expression levels to 62 and $80 \%$, respectively, of the levels seen in cells treated only with LPS. In contrast, a significant increase of mRNA expression of IL-10, a potent anti-inflammatory molecule, was observed in cells exposed to LPS and F-GWA + LPS treatments $(P<0.05)$ compared with untreated RAW264.7 cells (Figure 2C). However, the expression of IL-10 induced by LPS was not improved by F-GWA alone.

We also evaluated the effect of F-GWA treatment on the phosphorylation of proteins in the different MAPK pathways as a means of assessing the activation of these pathways. Lipopolysaccharide treatment of RAW264.7 cells increased the phosphorylation of both extracellular signal regulated kinase (ERK) and c-Jun N-terminal kinase (JNK), whereas the phosphorylation of p38 was not affected (Figure 3A). Pretreatment with F-GWA inhibited the phosphorylation of ERK and JNK at high concentrations $(1,000 \mu \mathrm{g} / \mathrm{mL})$, but it did not affect the phosphorylation of p38. Additionally, the phosphorylation of c-Jun in LPS-treated RAW264.7 cells was markedly inhibited by the F-GWA pretreatment in a dose-dependent manner (Figure 3B).
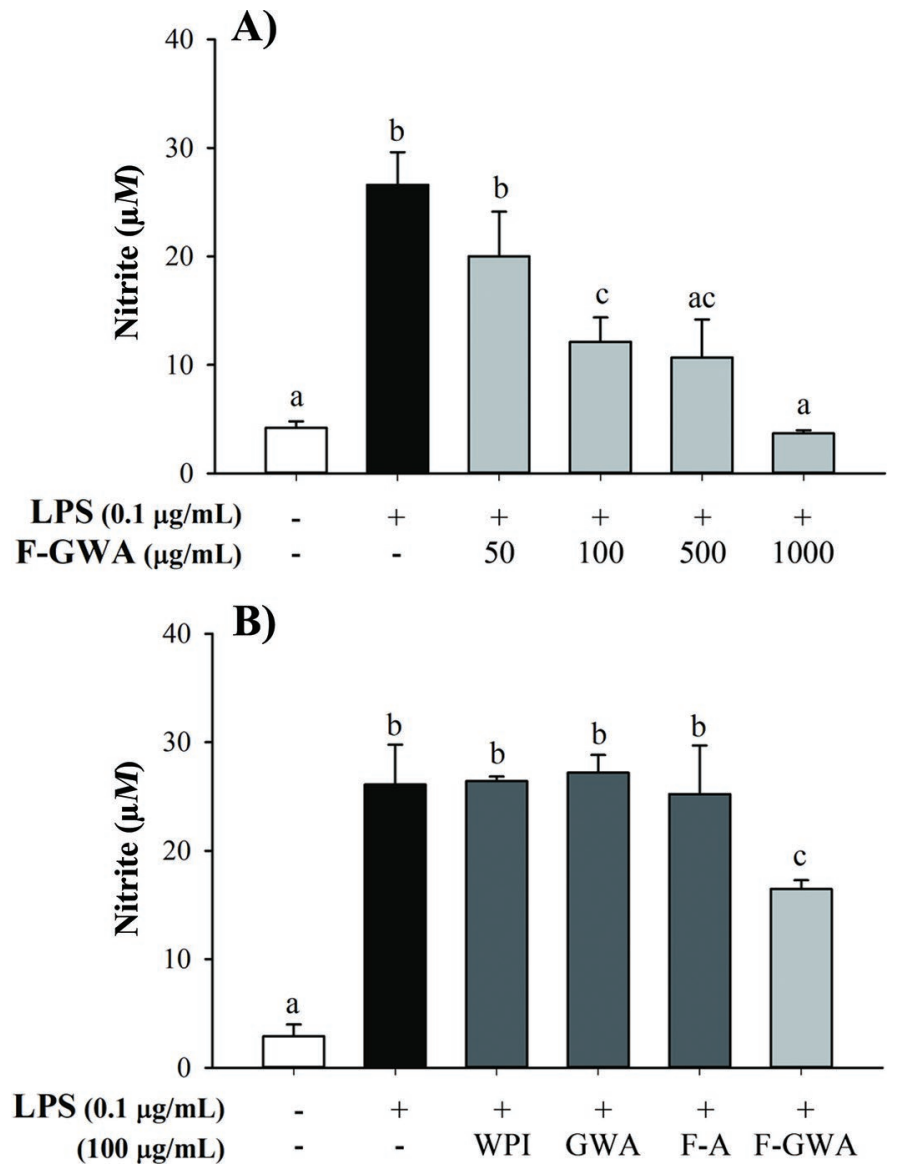

Figure 1. Reduction in nitric oxide (NO) production by the fermented glycated conjugate of whey protein isolate (WPI) and galactose (F-GWA) in RAW264.7 cells. The RAW264.7 cells were treated with LPS $(100 \mathrm{ng} / \mathrm{mL})$ for $16 \mathrm{~h}$ after they had been pretreated with the different concentrations of F-GWA for $2 \mathrm{~h}$. Nitric oxide production in RAW264.7 supernatants was measured using the Griess reagent. (A) Dose-dependent reduction in LPS-stimulated NO production by F-GWA pretreatment. (B) RAW264.7 cells were pretreated with 100 $\mu \mathrm{g} / \mathrm{mL}$ each of WPI, glycated conjugate of WPI and galactose (GWA), galactose fermented by Lactobacillus gasseri 4M13 (F-A), or F-GWA for $2 \mathrm{~h}$ as described above. Nitric oxide production in RAW264.7 supernatants was then measured using the Griess reagent. The different letters $(\mathrm{a}-\mathrm{c})$ denote a significant difference at $P<0.05$ using a 1 -way ANOVA with Tukey's multiple comparison test. The data are shown as the mean $\pm \mathrm{SD}$.

\section{Chemical Properties of F-GWA}

To examine the extent of glycation of GWA, its fluorescence intensity was measured. The fluorescence of GWA constantly increased during the glycation process, which occurred over a period of $24 \mathrm{~h}$ at $65^{\circ} \mathrm{C}$ and resulted in a fluorescence intensity that was 9-fold higher at $24 \mathrm{~h}$ than at $0 \mathrm{~h}$. The fluorescence of the WPI solution also doubled over $24 \mathrm{~h}$ (Figure $4 \mathrm{~A}$ ). In particular, the fluorescence of GWA rapidly increased for the first $18 \mathrm{~h}$ of the glycation process. The levels of ad- 


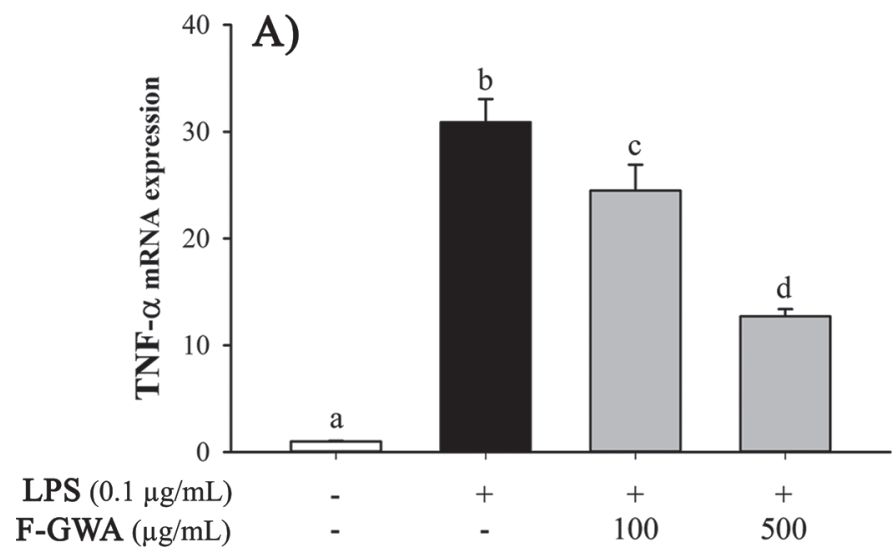

A)
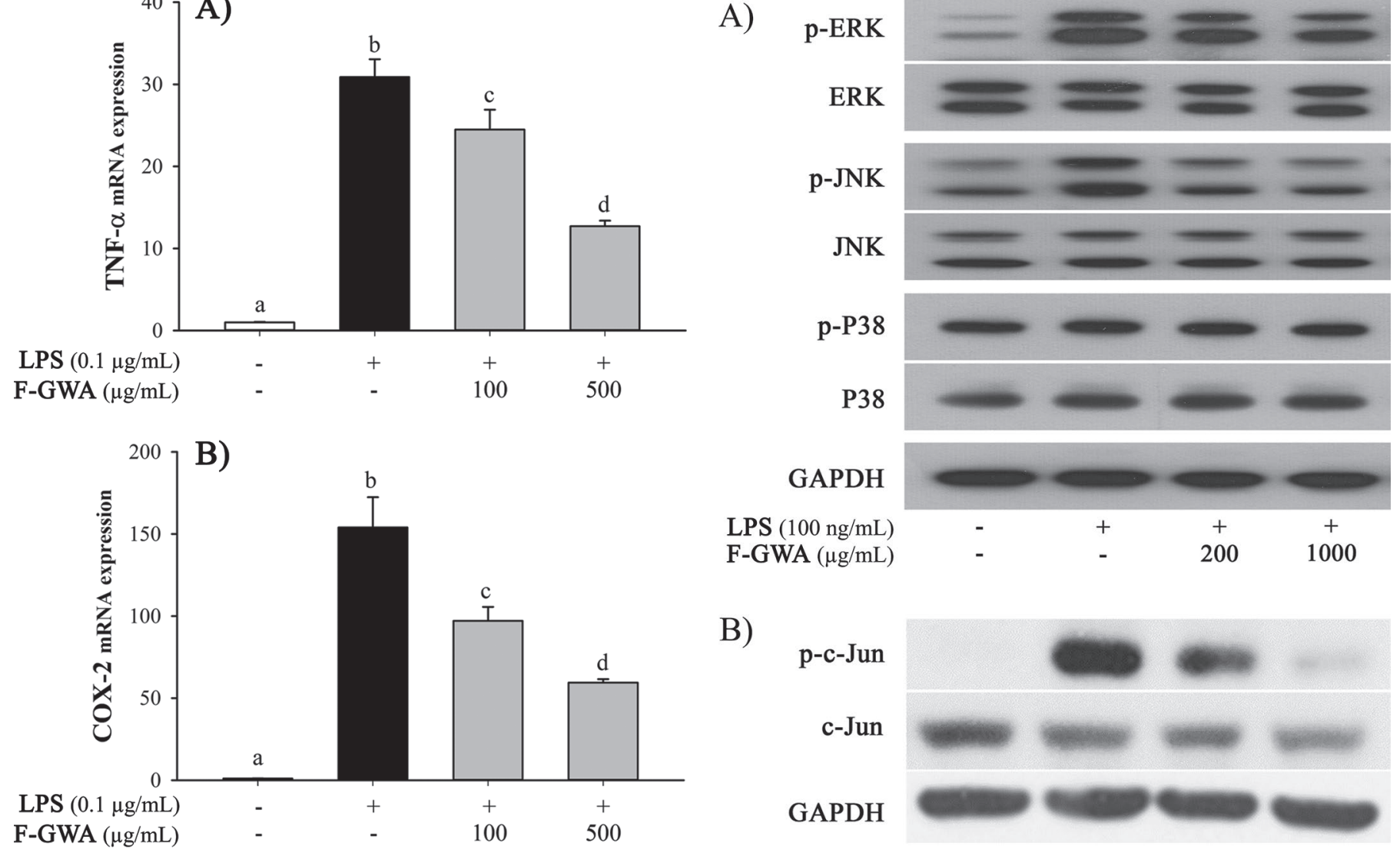

B)

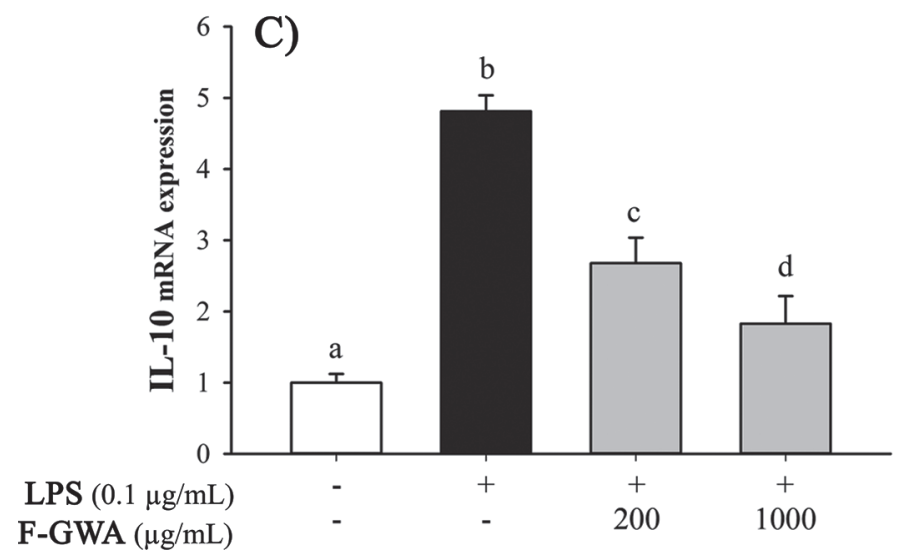

Figure 2. Reduction in the expression of LPS-induced inflammatory genes by the fermented glycated conjugate of whey protein isolate and galactose (F-GWA) in RAW264.7 cells. RAW264.7 cells were pretreated with different concentrations of F-GWA indicated for $2 \mathrm{~h}$, after which LPS (100 ng/mL) was added for a further $4 \mathrm{~h}$. The mRNA levels were then measured by quantitative reverse-transcription PCR. (A) Tumor necrosis factor- $\alpha$ (TNF- $\alpha$ ), (B) cyclooxygenase-2 (COX-2), and $(\mathrm{C}) \mathrm{IL}-10$. The expression levels were normalized using $\beta$-actin as the housekeeping gene and are reported as the fold-change relative to the control (untreated) group. The different letters (a-d) denote a significant difference at $P<0.05$ using a 1-way ANOVA with Tukey's multiple comparison test. The data are shown as the mean $\pm \mathrm{SD}$.

\section{p-c-Jun \\ p-c-Jun \\ c-Jun \\ GAPDH \\ LPS $(100 \mathrm{ng} / \mathrm{mL})$ \\ F-GWA $(\mu \mathrm{g} / \mathrm{mL})$}

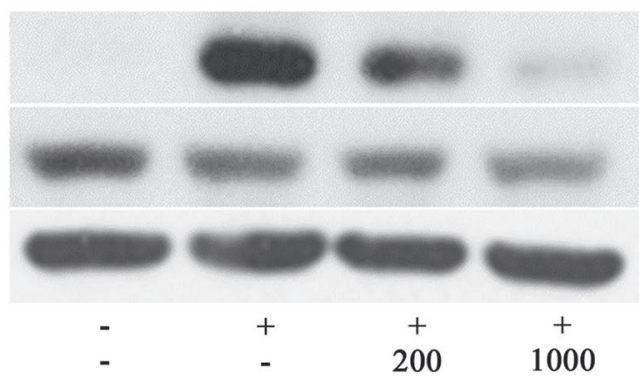

Figure 3. Reduction in LPS-stimulated ERK (extracellular signalregulated kinase), JNK (c-Jun N-terminal kinase), and c-Jun phosphorylation (p-) by the fermented glycated conjugate of whey protein isolate and galactose (F-GWA) in RAW264.7 cells. The RAW264.7 cells were pretreated with the different concentrations of F-GWA indicated for $2 \mathrm{~h}$, after which LPS $(100 \mathrm{ng} / \mathrm{mL})$ was added for a further 30 min. The levels of phosphorylation of the indicated mitogen-activated protein kinases (MAPK) proteins (A) and c-Jun (B) were assessed by Western blotting of cell lysates using the respective antibodies. GAPDH was used as the housekeeping protein.

vanced Maillard products were detected by measuring the fluorescence of these products following excitation at $330 \mathrm{~nm}$ and emission at $420 \mathrm{~nm}$ (Guan et al., 2005; Figure 4B). Fluorescent advanced Maillard products also constantly increased for the first $18 \mathrm{~h}$ of the glycation reaction at $65^{\circ} \mathrm{C}$, after which the levels decreased slightly. The concentrations of furosine, which is a major marker of the early stages of the Maillard reaction (Erbersdobler and Somoza, 2007), increased steadily over the 24-h period and were 12.7 fold higher at $24 \mathrm{~h}$ compared with $0 \mathrm{~h}$ (Figure 4C).

After the glycation process, we characterized the glycation sites in WPI using MALDI-TOF MS/MS analysis (Table 1 ). From the results, only $\beta-\mathrm{LG}$, a WPI protein, participated in the glycation reaction with 

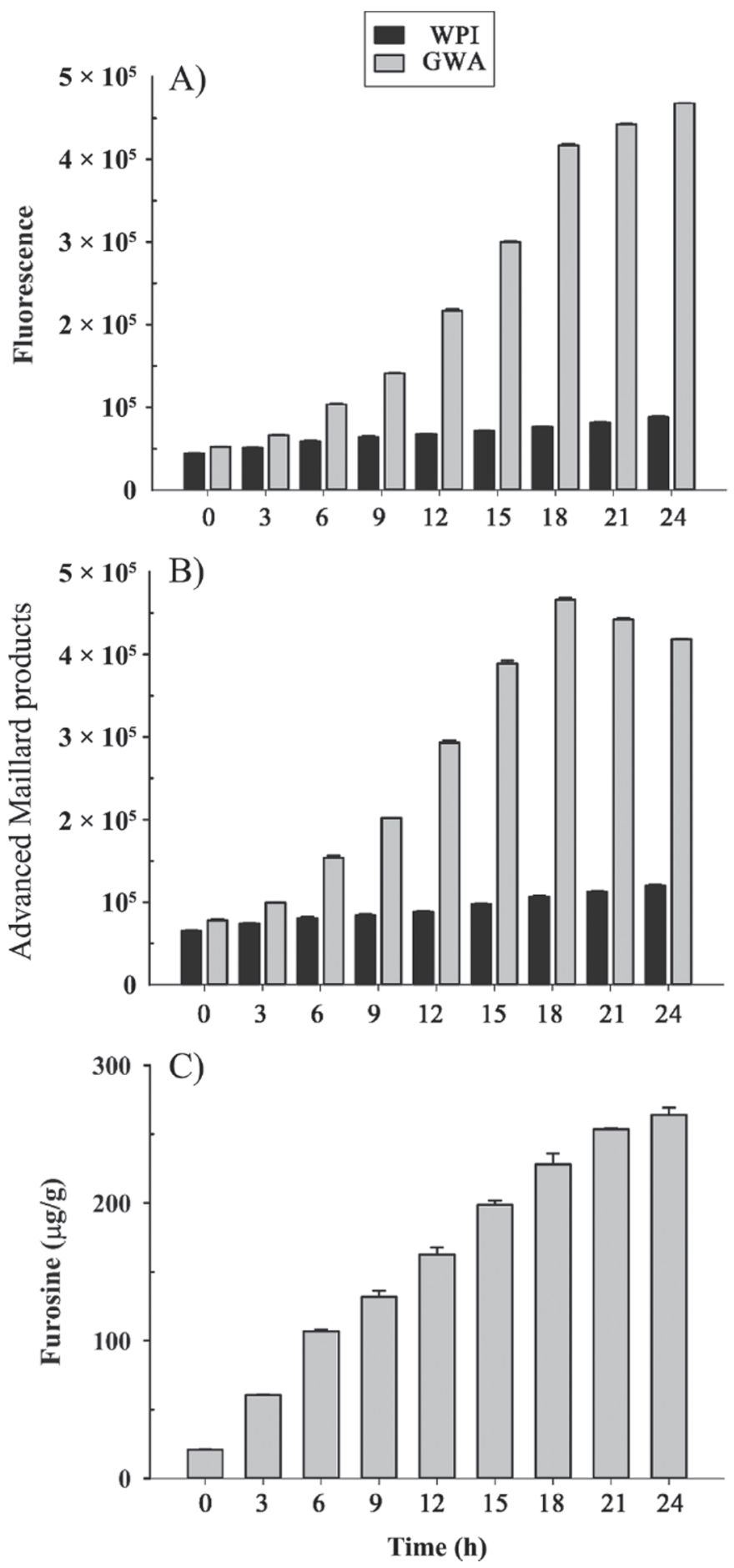

Figure 4. Characterization of the glycated conjugate of whey protein isolate (WPI) and galactose (GWA). Over $24 \mathrm{~h}$ of the glycation process, WPI and GWA solutions were collected every $3 \mathrm{~h}$ from 0 to 24 $\mathrm{h}$. (A) Increase in fluorescence intensity during the glycation process. Fluorescence was measured at an excitation wavelength of $370 \mathrm{~nm}$ and an emission wavelength of $440 \mathrm{~nm}$. (B) The increase in fluorescence derived from advanced Maillard products was measured at an excitation wavelength of $330 \mathrm{~nm}$ and an emission wavelength of $420 \mathrm{~nm}$. (C) Increases in the $\varepsilon-N$-2-furoylmethyl-L-lysine (furosine) concentration in GWA were measured using HPLC. The data are shown as the mean $\pm \mathrm{SD}$ galactose. Lysine $\mathrm{K}^{157}$ present in the ${ }^{155}$ ALKALPMHIR $^{164}$ peptide obtained from digested $\beta$-LG was found to form an adduct with hexose and was detected at $m / z 1$,311. In addition, $\mathrm{K}^{117}$ present in the ${ }^{117}$ KYLLFCMENSAEPEQSLACQCLVR ${ }^{140}$ peptide obtained from digested $\beta$-LG was also found to form an adduct with hexose and was detected at $m / z 3,108$ and 3,136. In addition, methionines $\mathrm{M}^{40}$ and $\mathrm{M}^{123}$ were found to be oxidized, and asparagine $\mathrm{N}^{125}$ and glutamine $\mathrm{Q}^{131}$ were found to be sites for deamination in GWA.

We also compared the organic acid concentration changes resulting from the fermented products (Table 2 ). Although both solutions contained the same concentrations of protein and galactose, F-GWA contained more organic acids than F-WA because glycation before fermentation produced acetic acid and propionic acid. The glycation process before fermentation also increased the levels of formic acid and lactic acid by 44 and $182 \%$, respectively, compared with no glycation.

Before its use, GWA was subjected to ultrafiltration; consequently, the increase in the levels of both acids in F-GWA was primarily due to the GWA fermentation process. From this analysis, not only do the glycation and fermentation processes each increase the concentration of acids, but glycation followed by fermentation leads to a synergistic increase in the acid concentrations in F-GWA.

\section{DISCUSSION}

Recently, Cardoso et al. (2018) reported that the Maillard reaction rate of $\alpha-\mathrm{LA}$ and $\beta$-LG with galactose in whey is almost twice that with lactose. In the present study, we chose galactose as the sugar source to react with whey protein in order to minimize the influence of milk lactose and better characterize the anti-inflammatory compounds present in F-GWA as well as the mechanisms of anti-inflammatory activities.

To confirm the anti-inflammatory effect of F-GWA, we assessed the inflammatory response of cells exposed to it by measuring the mRNA expression of inflammatory response genes. Macrophages treated with LPS drastically increase TNF- $\alpha$ gene expression. Most cells can produce $\mathrm{TNF}-\alpha$ in response to an inflammatory signal, but monocytes produce TNF- $\alpha$ as a quick response following pathogen recognition (Lin and Yeh, 2005). The production of TNF- $\alpha$ can activate inflammatory cells in surrounding tissue and promote the production of pro-inflammatory molecules such as prostaglandins (Parameswaran and Patial, 2010). Prostaglandins are generated from arachidonate by cyclooxygenase in response to an inflammatory signal (Ricciotti and FitzGerald, 2011). Consequently, the levels of TNF- $\alpha$ and secreted prostaglandins can serve 
Table 1. Modified peptide profiles of glycated conjugate of whey protein isolate and galactose (GWA) using MALDI-TOF MS/MS

\begin{tabular}{|c|c|c|c|}
\hline Protein & Residue & Amino acid sequence $^{1}$ & Modification \\
\hline
\end{tabular}

${ }^{1}$ Modification sites in the protein are shown underlined in bold.

${ }^{2}$ The modification site in the protein is glycated with hexose.

as indicators of the severity of inflammation. In this study, F-GWA pretreatment of macrophages reduced the LPS-stimulated increase in the gene expression levels of both TNF- $\alpha$ and COX-2. The innate immune response is stimulated by the recognition of pathogens by cells, particularly immune cells (Plüddemann et al., 2011). Lipopolysaccharide represents one of the most widely known pathogen-associated molecular patterns and is found within the gram-negative bacterial cell wall. Upon exposure, the immune system recognizes LPS via toll-like receptor 4 (TLR4), which activates the host defense responses, with or without MyD88 as a signaling adapter (Lu et al., 2008). The MyD88-dependent pathway leads to the activation of the MAPK pathway. This activation, which can be monitored by measuring the phosphorylation levels of MAPK, leads to the induction of transcription factor activator protein 1 (AP-1) (Kawai and Akira, 2006; Lu et al., 2008). Further, TLR4 activated by LPS eventually activates the transcription factors nuclear factor $(\mathbf{N F})-\kappa \mathrm{B}$ and AP-1, which are downstream of the MyD88-dependent pathway, increasing the transcription of pro-inflammatory cytokines ( $\mathrm{Lu}$ et al., 2008). In the present study, the phosphorylation of p38 was not induced by LPS. Xiao et al. (2002) reported that p38 phosphorylation in RAW264.7 cells treated with LPS $(100 \mu \mathrm{g} / \mathrm{mL})$ at various time points was detected at 5 min and reached a maximum at $15 \mathrm{~min}$; from $30 \mathrm{~min}$ to $60 \mathrm{~min}$, the protein expression fell sharply to the control level. Although we did not confirm the possibility, the lack of changes in p38 phosphorylation induced by LPS treatment in our study likely resulted from the relatively longer LPS treatment time $(2 \mathrm{~h})$ for RAW264.7 cells. However, F-GWA was shown to significantly inhibit the phosphorylation of ERK, JNK, and c-Jun in response to the inflammatory LPS signal, which ultimately suppressed the LPS-induced increases in the gene expression levels of TNF- $\alpha$ and COX-2. In contrast, IL-10 is a major anti-inflammatory cytokine that is expressed by macrophages and myeloid dendritic cells, and activation of the ERK pathway in these cells leads to IL-10 induction (Saraiva and O'Garra, 2010). Those authors demonstrated that inhibition of both the ERK and p38 pathways in LPS-stimulated macrophages results in almost complete elimination of IL-10 production. We found that F-GWA reduced IL-10 gene expression after LPS treatment, and we note that inhibition of ERK phosphorylation by F-GWA pretreatment would lead to IL-10 inhibition in LPS challenge.

Considerable overlap occurs, including downstream gene activation, activation by the same extracellular stimulation, and MAPK-induced activation of JNK between NF- $\kappa \mathrm{B}$ and AP-1 in LPS-challenged macrophages (Tugal et al., 2013). In this study, F-GWA clearly inhibited the ERK and JNK phosphorylation caused by LPS treatment and inhibited c-Jun phosphorylation, contributing to increased AP-1 activation, caused by LPS treatment. On the other hand, after LPS treatment for $30 \mathrm{~min}, \mathrm{NF}-\kappa \mathrm{B}$ p65 was slightly increased in the nuclear fraction (Supplemental Figure S2; https://doi.org/10.3168/jds.2018-15774). Pretreatment with F-GWA at $200 \mu \mathrm{g} / \mathrm{mL}$ for $2 \mathrm{~h}$ before LPS treatment enhanced NF- $\kappa \mathrm{B}$ p65 expression; however, pretreatment with a higher concentration of F-GWA

Table 2. Organic acid concentrations $(\mu \mathrm{g} / \mathrm{g})$

\begin{tabular}{lrccc}
\hline Sample $^{1}$ & Formic acid & Acetic acid & Propionic acid & Lactic acid \\
\hline WPI & - & - & - & - \\
F-WA & $61.36 \pm 6.14$ & - & - & $1,294.59 \pm 21.75$ \\
GWA & $107.89 \pm 2.66$ & $26.13 \pm 2.63$ & $157.36 \pm 3.41$ & $2,364.62 \pm 61.60$ \\
F-GWA & $88.63 \pm 3.63$ & $35.86 \pm 0.16$ & $680.87 \pm 7.85$ & $3,650.50 \pm 164.42$ \\
\hline
\end{tabular}

${ }^{1} \mathrm{WPI}=$ intact whey protein isolate; F-WA $=$ fermented WPI and galactose by Lactobacillus gasseri $4 \mathrm{M} 13$; GWA $=$ glycated WPI and galactose; and F-GWA $=$ fermented GWA with L. gasseri 4M13. The data are shown as the mean $\pm \mathrm{SD}$. 
$(1,000 \mu \mathrm{g} / \mathrm{mL})$ inhibited NF- $\mathrm{B}$ p65 expression. Under our experimental condition, LPS did not appear to contribute to NF- $\kappa \mathrm{B}$ activation.

The MAPK/AP-1 pathway is linked not only to the production of cytokines and enzymes, but also to the regulation of NO production through the transcription of inducible NO synthase in macrophages (Medzhitov, 2001; Shaheen and Corbett, 2015). Nitric oxide is produced by inducible NO synthase as part of the innate immune response, and it has antimicrobial activity that damages other cells (e.g., bacteria and tumor cells; Bogdan, 2001). Pretreatment of RAW264.7 cells with F-GWA decreased LPS-stimulated NO production in a dose-dependent manner. These results suggest that F-GWA treatment of cells inhibits the phosphorylation of ERK and JNK from the MAPK family and subsequently the LPS-induced inflammatory response.

A comparison of the effects of glycation and fermentation by $L$. gasseri $4 \mathrm{M} 13$ on the anti-inflammatory effect is shown in Figure 1B. Importantly, only the sample that was both glycated and fermented showed an anti-inflammatory effect; separately, neither process was associated with this effect. Therefore, the 2 processes synergistically provided an anti-inflammatory property to F-GWA. This result led us to examine the chemical changes that occur during each process. First, to confirm the glycation-related changes during the reaction of WPI and galactose, we analyzed increases in fluorescence and changes in the furosine concentration of the reactant solutions over time. Because many unidentified Maillard reaction products are fluorescent, the extent of the Maillard reaction is generally estimated by measuring fluorescence (van Boekel, 2001). As shown in Figure 4, the fluorescence representing advanced Maillard products declined after $18 \mathrm{~h}$, whereas the general fluorescence and furosine concentration increased. Furosine is an acid hydrolysate form of an Amadori product (Erbersdobler and Somoza, 2007); therefore, from the increase in furosine during incubation, reversible Amadori products were produced during the incubation of WPI and galactose. This outcome suggests that the initial stage of Maillard reaction occurred during the WPI and galactose incubation. Additionally, to confirm glycation between WPI and galactose, we investigated the glycation of WPI-derived peptides using MALDI-TOF-MS/MS. We confirmed that $\mathrm{K}^{117}$ and $\mathrm{K}^{157}$ from $\beta$-LG were glycated with galactose; these 2 lysine residues are well-known glycation sites in $\beta-\mathrm{LG}$ (Dyer et al., 2016; Oh et al., 2016).

As shown in Table 2, the glycated conjugate of WPI and galactose produce more organic acids than the nonglycated WPI and galactose during the fermentation process. Because formic acid and acetic acid are degradation products of the Maillard reaction (van Boekel, 2001), GWA contained higher levels of formic acid than other fermented products. Corzo-Martínez et al. (2012) reported that galactosylated $\beta-L G$ could enhance the growth of Lactobacillus better than lactosylated $\beta-L G$, and they also showed that galactosylated casein and $\beta-\mathrm{LG}$ could produce tagatosyllysine, an Amadori rearrangement product. Tagatose is an isomer of galactose, which can be used by Lactobacillus as a prebiotic (Bertelsen et al., 1999; Corzo-Martínez et al., 2008). It should be noted that acetic acid and propionic acid, which greatly increased during glycation before fermentation, are the most characteristic fermentation organic acids of F-GWA in addition to lactic acid in this study. These short-chain fatty acids (SCFA) are major fermentation product of anaerobic bacteria. As L. gasseri 4M13 isolated from feces of healthy infants, the lactic acid bacteria could produce SCFA through GWA fermentation. Those SCFA from fermentation are well known for their anti-inflammatory effects through TLR4/MAPK inhibition (Li et al., 2018) and inhibition of TNF- $\alpha$ and PGE2 (Corrêa-Oliveira et al., 2016), whose production is stimulated by LPS. Previously, bacterial fermented SCFC in Nipa vinegar were shown to have a positive effect on inflammation and lipid metabolism in obese mice, compared with synthetic SCFA (Beh et al., 2017), Therefore, these common SCFA produced by fermentation of GWA could act as major inducers of the anti-inflammatory effect, which is in line with the NO inhibition results mainly found with the F-GWA treatment in our study. Therefore, WPI and galactose are more suitable for fermentation with L. gasseri $4 \mathrm{M} 13$ due to the Maillard reaction, and this combination leads to an enhanced fermentation rate compared with nonglycated WPI and galactose. Furthermore, the reduction in NO production in macrophages was greater with F-GWA treatment than with other samples, which could have been related to the fermentation efficiency of F-GWA. In conclusion, F-GWA has an anti-inflammatory effect because it reduces NO production and downregulates TNF- $\alpha$ and COX-2 gene expression by inhibiting the phosphorylation of ERK and JNK in response to LPS-induced inflammation in RAW264.7 cells.

\section{ACKNOWLEDGMENTS}

This research was supported by the High ValueAdded Food Technology Development Program (115006-03-HD030), Korea Institute of Planning and Evaluation for Technology in Food, Agriculture, Forestry and Fisheries (iPET), National Research Foundation of Korea grant funded by the Korean government (No. 2017R1A2B4012182), and School of Life Sciences 
\& Biotechnology of Korea University for BK21PLUS. The authors also thank the Institute of Biomedical Science \& Food Safety, CJ-Korea Food Safety Hall (Seoul, South Korea), for providing the equipment and facilities.

\section{REFERENCES}

Beh, B. K., N. E. Mohamad, S. K. Yeap, H. Ky, S. Y. Boo, J. Y. H. Chua, S. W. Tan, W. Y. Ho, S. A. Sharifuddin, K. Long, and N. B. Alitheen. 2017. Anti-obesity and anti-inflammatory effects of synthetic acetic acid vinegar and Nipa vinegar on high-fat-dietinduced obese mice. Sci. Rep. 7:6664-6672.

Bertelsen, H., B. B. Jensen, and B. Buemann. 1999. D-Tagatose-A novel low-calorie bulk sweetener with prebiotic properties. Pages 98-109 in Low-Calories Sweeteners: Present and Future. Vol. 85. C. A, ed. Karger Publishers, Barcelona, Spain.

Bogdan, C. 2001. Nitric oxide and the immune response. Nat. Immunol. 2:907-916.

Cardoso, H. B., P. A. Wierenga, H. Gruppen, and H. A. Schols. 2018. Maillard induced glycation behavior of individual milk proteins. Food Chem. 252:311-317.

Chatterton, D. E. W., D. N. Nguyen, S. B. Bering, and P. T. Sangild. 2013. Anti-inflammatory mechanisms of bioactive milk proteins in the intestine of newborns. Int. J. Biochem. Cell Biol. 45:1730-1747.

Chawla, S. P., R. Chander, and A. Sharma. 2009. Antioxidant properties of Maillard reaction products obtained by gamma-irradiation of whey proteins. Food Chem. 116:122-128.

Chun, S.-H., H. A. Lee, K. B. Lee, S. H. Kim, K.-Y. Park, and K.W. Lee. 2016. Effects of glycated whey protein concentrate on pro-inflammatory cytokine expression and phagocytic activity in RAW264.7 macrophages. Biol. Pharm. Bull. 39:199-206.

Corrêa-Oliveira, R., J. L. Fachi, A. Vieira, F. T. Sato, and M. A. R. Vinolo. 2016. Regulation of immune cell function by short-chain fatty acids. Clin. Transl. Immunology 5:e73-e80.

Corzo-Martínez, M., M. Ávila, F. J. Moreno, T. Requena, and M. Villamiel. 2012. Effect of milk protein glycation and gastrointestinal digestion on the growth of bifidobacteria and lactic acid bacteria. Int. J. Food Microbiol. 153:420-427.

Corzo-Martínez, M., F. J. Moreno, A. Olano, and M. Villamiel. 2008. Structural characterization of bovine $\beta$-lactoglobulin-galactose/ tagatose Maillard complexes by electrophoretic, chromatographic, and spectroscopic methods. J. Agric. Food Chem. 56:4244-4252.

Da Silva, M. S., and I. Rudkowska. 2015. Dairy nutrients and their effect on inflammatory profile in molecular studies. Mol. Nutr. Food Res. 59:1249-1263.

Donkor, O. N., A. Henriksson, T. Vasiljevic, and N. P. Shah. 2005. Probiotic strains as starter cultures improve angiotensin-converting enzyme inhibitory activity in soy yogurt. J. Food Sci. 70:375-381.

Dyer, J. M., A. Grosvenor, A. Thomas, C. Callaghan, S. Deb-Choudhury, and S. Haines. 2016. Proteomic tracking of hydrothermal Maillard and redox modification in lactoferrin and $\beta$-lactoglobulin: Location of lactosylation, carboxymethylation, and oxidation sites. J. Dairy Sci. 99:3295-3304.

Erbersdobler, H. F., and V. Somoza. 2007. Forty years of furosineForty years of using Maillard reaction products as indicators of the nutritional quality of foods. Mol. Nutr. Food Res. 51:423-430.

Guan, R.-F., D.-H. Liu, X.-Q. Ye, and K. Yang. 2005. Use of fluorometry for determination of skim milk powder adulteration in fresh milk. J. Zhejiang Univ. Sci. B 6:1101-1106.

Kang, M., N. S. Oh, M. Kim, H. Y. Ahn, H. J. Yoo, M. Sun, S.-H. Kang, H. J. Yang, D. Y. Kwon, and J. H. Lee. 2017. Supplementation of fermented Maillard-reactive whey protein enhances immunity by increasing NK cell activity. Food Funct. 8:1718-1725.

Kawai, T., and S. Akira. 2006. TLR signaling. Cell Death Differ. 13:816-825.

Li, M., B. C. van Esch, G. T. Wagenaar, J. Garssen, G. Folkerts, and P. A. Henricks. 2018. Pro- and anti-inflammatory effects of short chain fatty acids on immune and endothelial cells. Eur. J. Pharmacol. 831:52-59.

Lin, W.-J., and W.-C. Yeh. 2005. Implication of Toll-like receptor and tumor necrosis factor $\alpha$ signaling in septic shock. Shock 24:206209.

Livak, K. J., and T. D. Schmittgen. 2001. Analysis of relative gene expression data using real-time quantitative PCR and the $2^{-\triangle \Delta C T}$ method. Methods 25:402-408.

Lu, Y.-C., W.-C. Yeh, and P. S. Ohashi. 2008. LPS/TLR4 signal transduction pathway. Cytokine 42:145-151.

Marshall, K. 2004. Therapeutic applications of whey protein. Altern. Med. Rev. 9:136-156.

Masood, M. I., M. I. Qadir, J. H. Shirazi, and I. U. Khan. 2011. Beneficial effects of lactic acid bacteria on human beings. Crit. Rev. Microbiol. 37:91-98.

Matar, C., J. C. Valdez, M. Medina, M. Rachid, and G. Perdigon. 2001. Immunomodulating effects of milks fermented by Lactobacillus helveticus and its non-proteolytic variant. J. Dairy Res. 68:601-609.

Medzhitov, R. 2001. Toll-like receptors and innate immunity. Nat. Rev. Immunol. 1:135-145.

Merenstein, D., M. Murphy, A. Fokar, R. K. Hernandez, H. Park, H. Nsouli, M. E. Sanders, B. A. Davis, V. Niborski, F. Tondu, and N. M. Shara. 2010. Use of a fermented dairy probiotic drink containing Lactobacillus casei (DN-114 001) to decrease the rate of illness in kids: The DRINK study A patient-oriented, double-blind, cluster-randomized, placebo-controlled, clinical trial. Eur. J. Clin. Nutr. 64:669-677.

Oh, N. S., J. Y. Joung, J. Y. Lee, and Y. Kim. 2018. Probiotic and anti-inflammatory potential of Lactobacillus rhamnosus 4B15 and Lactobacillus gasseri 4M13 isolated from infant feces. PLoS One 13:e0192021.

Oh, N. S., J. Y. Joung, J. Y. Lee, Y. Kim, and S. H. Kim. 2017. Enhancement of antioxidative and intestinal anti-inflammatory activities of glycated milk casein after fermentation with Lactobacillus rhamnosus 4B15. J. Agric. Food Chem. 65:4744-4754.

Oh, N. S., H. A. Lee, J. Y. Lee, J. Y. Joung, K. B. Lee, Y. Kim, K. W. Lee, and S. H. Kim. 2013. The dual effects of Maillard reaction and enzymatic hydrolysis on the antioxidant activity of milk proteins. J. Dairy Sci. 96:4899-4911.

Oh, N. S., J. Y. Lee, H. A. Lee, J. Y. Joung, Y. K. Shin, S. H. Kim, Y. Kim, and K. W. Lee. 2016. Chemical characteristics and enhanced hepatoprotective activities of Maillard reaction products derived from milk protein-sugar system. J. Dairy Sci. 99:947-958.

Panesar, P. S., J. F. Kennedy, C. J. Knill, and M. Kosseva. 2010. Production of $\mathrm{L}(+)$ lactic acid using Lactobacillus casei from whey. Braz. Arch. Biol. Technol. 53:219-226.

Parameswaran, N., and S. Patial. 2010. Tumor necrosis factor- $\alpha$ signaling in macrophages. Crit. Rev. Eukaryot. Gene Expr. 20:87-103.

Pescuma, M., E. M. Hébert, F. Mozzi, and G. F. D. Valdez. 2010. Functional fermented whey-based beverage using lactic acid bacteria. Int. J. Food Microbiol. 141:73-81.

Plüddemann, A., S. Mukhopadhyay, and S. Gordon. 2011. Innate immunity to intracellular pathogens: Macrophage receptors and responses to microbial entry. Immunol. Rev. 240:11-24.

Prasad, S., K. Srikanth, A. M. Limaye, and S. Sivaprakasam. 2014. Homo-fermentative production of d-lactic acid by Lactobacillus sp. employing casein whey permeate as a raw feed-stock. Biotechnol. Lett. 36:1303-1307.

Pyo, M. C., S.-Y. Yang, S.-H. Chun, N. S. Oh, and K.-W. Lee. 2016. Protective effects of Maillard reaction products of whey protein concentrate against oxidative stress through an Nrf2-dependent pathway in HepG2 cells. Biol. Pharm. Bull. 39:1437-1447.

Ricciotti, E., and G. A. FitzGerald. 2011. Prostaglandins and inflammation. Arterioscler. Thromb. Vasc. Biol. 31:986-1000.

Saraiva, M., and A. O'Garra. 2010. The regulation of IL-10 production by immune cells. Nat. Rev. Immunol. 10:170-181.

Shaheen, Z. R., and J. A. Corbett. 2015. Macrophage expression of inflammatory genes in response to EMCV infection. Biomolecules 5:1938-1954. 
Tugal, D., X. Liao, and M. K. Jain. 2013. Transcriptional control of macrophage polarization. Arterioscler. Thromb. Vasc. Biol. 33:1135-1144.

van Boekel, M. A. J. S. 1998. Effect of heating on Maillard reactions in milk. Food Chem. 62:403-414.

van Boekel, M. A. J. S. 2001. Kinetic aspects of the Maillard reaction: A critical review. Nahrung 45:150-159.

Vinderola, G., G. Perdigon, J. Duarte, D. Thangavel, E. Farnworth, and C. Matar. 2006. Effects of kefir fractions on innate immunity. Immunobiology 211:149-156.

Wang, W. Q., Y. H. Bao, and Y. Chen. 2013. Characteristics and antioxidant activity of water-soluble Maillard reaction products from interactions in a whey protein isolate and sugars system. Food Chem. 139:355-361.

Xiao, Y. Q., K. Malcom, G. S. Worthen, S. Gardai, W. P. Schiemann, V. A. Fadok, D. L. Bratton, and P. M. Henson. 2002. Cross-talk between ERK and p38 mediates selective suppression of pro-inflammatory cytokines by transforming growth factor- $\beta$. J. Biol. Chem. 277:14884-14893.

Yamaguchi, M., K. Yoshida, and M. Uchida. 2009. Novel functions of bovine milk-derived $\alpha$-lactalbumin: Anti-nociceptive and antiinflammatory activity caused by inhibiting cyclooxygenase- 2 and phospholipase A2. Biol. Pharm. Bull. 32:366-371. 